\title{
COMPUTER SIMULATION OF ELECTRON-FLOW BUNCHING IN THE PULSAR MAGNETOSPHERE
}

\author{
Yu. A. RYLov \\ Institute for Problems in Mechanics, Academy of Sciences
}

\section{Introduction}

Bunch curvature emission is one of the well known pulsar radio emission mechanisms. The problem of bunch formation is very important for understanding the pulsar radio emission mechanism. In the axisymmetric pulsar magnetospheric bunching arises in the outflow channel as a result of interaction between moving electrons (DP) and captured ones (SP) (Rylov 1988). A numerical simulation was undertaken to determine how strongly the electron flow is bunched. The bunching appears to be very strong. It can be treated as a gas of electron bunches rather than small fluctuations of the electron tow. The thermal radio emission of the electron-bunch gas has a very high brightness temperature and sharp directivity. The power consumed in electron-bunch gas heating is sufficient to explain the pulsar radio emission. The pulsar radio emission mechanism appears to be thermal (the electron bunches move chaotically) and coherent (electrons of the bunch emit coherently) at the same time. For this reason the radio emission mechanism is very stable.

\section{Spherically symmetric model of the outflow channel}

According to the pulsar magnetospheric model (Rylov 1985) electrons move in the pulsar magnetosphere along the outflow channel from the pole to a region of lower latitude. Depending on the value of some parameter $\alpha$ which describes the inhomogeneity of the background charge, the electron flow inside the outflow channel is either stable or not. The physical significance of the non-uniformity parameter $\alpha$ is described in papers by Rylov (1979, 1987). Here we note only that if $\alpha<\alpha_{c}$, then the electron flow is stable [dynamical phase (DP) only]. If $\alpha>\alpha_{c}$, then, besides the DP, some captured electrons [static phase (SP)] arise inside the outflow channel (Rylov 1979). The dynamical phase moves through the SP, and a two-beam instability arises. This leads to irregular non-stationary electron flow inside the outflow channel.

This electron motion was simulated numerically in the simple spherically symmetric case, when elec- trons move along the radius in the self-consistent field. (The magnetic field is supposed to be fixed and radial). In the course of the calculation all physical quantities (energy density of the electric field $E^{2} / 2$, potential $\Phi$, electrical current $\mathrm{j}$, power $j \Phi$, Lorentz factor $\gamma$, and charge density $\rho$ ) were averaged over time (Rylov 1987). As a result the mean values of these quantities as functions of the parameter $\alpha$ were obtained. Here only the averaged value of the Lorentz factor $\gamma$ is presented (figure 1).

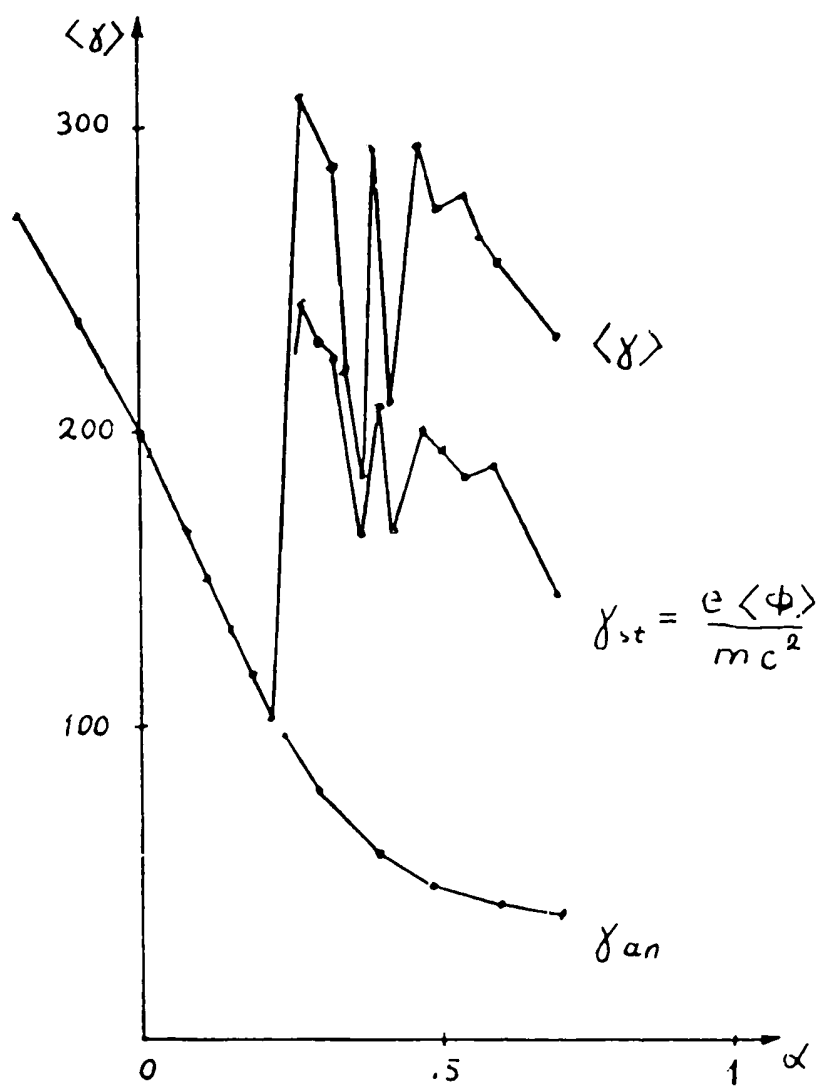

Figure 1 The Lorentz factor at the end of the outflow channel as a function of the non-uniformity parameter $\alpha . \gamma$ is the Lorentz factor of the stationary flow.

Investigation of these functions shows that their form changes sharply at the critical value $\alpha_{c}=0.25$ of the parameter $\alpha$. The results can be treated in the sense that at $\alpha>\alpha_{c}$ the electron motion can be considered as the motion of a gas consisting of electron bunches. The mean spatial spectrum of the charge density (figure 2) at $\alpha=0.3>\alpha_{c}=0.25$ and 
at $\alpha=0.2<\alpha_{\mathrm{c}}$ confirms this interpretation. One can see in figure 2 that there are bunches of all sizes inside the outflow channel at $\alpha>\alpha_{\mathrm{c}}$.

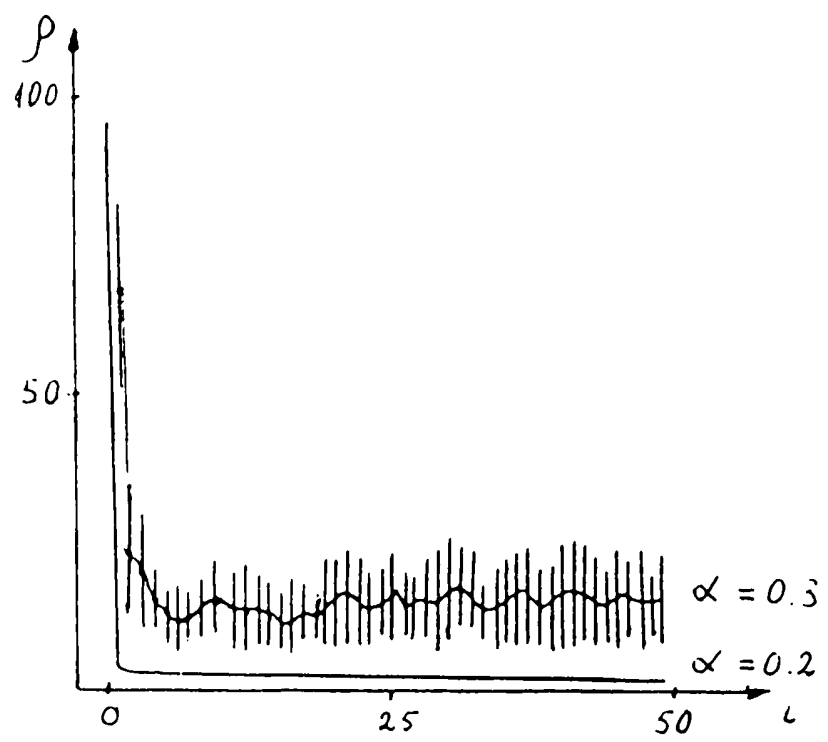

Figure 2 The mean spatial spectrum of the charge density ( $i$ is the number of the harmonic) is its magnitude. The vertical lines show the mean value of the fluctuation.

The mean Lorentz factor $\langle\gamma\rangle$ at the end of the outflow channel can be represented as a sum

$$
\langle\gamma\rangle=\gamma_{\mathbf{s t}}+\gamma_{\mathbf{f l}},
$$

where $\gamma_{\mathbf{s t}}$ is the Lorentz factor describing the electron acceleration in the mean potential $\langle\Phi\rangle, \gamma_{\mathrm{st}}=$ $e\langle\Phi\rangle / m c^{2}$, and $\gamma_{\mathrm{fl}}$ is the Lorentz factor describing the energy of the thermal motion of the electron bunches. The dependence of the Lorentz factors $\gamma_{\mathrm{st}}$ and $\gamma_{\mathrm{f}}$ on the extracting charge $Q_{\mathrm{m}}$ and on the relative length, $a$, of the outflow channel is shown in figures 3 and 4 . The extracting charge $Q_{m}$ is proportional to the extracting electrical field at the end of the outflow channel.

One can conclude that $\gamma_{\mathbf{f}}$ is approximately proportional to the length $a$ of the outflow channel, and it does not depend on $Q_{\mathrm{m}}$. It appears that the "heating" of the outflow channel is like the usual heating of an ohmic resistance. Extrapolation of this circumstance to the outflow channel of the axisymmetric model of the pulsar magnetosphere (Rylov 1977, 1985) with magnetic field $H_{\mathrm{p}}=10^{12} \mathrm{G}$ and period $P=1 \mathrm{~s}\left(R=10^{6} \mathrm{~cm}\right)$ leads to the following power consumed for heating of the outflow channel (Rylov 1987)

$$
\frac{\partial \varepsilon}{\partial t} \approx 10^{28.5} \mathrm{erg} \mathrm{s}^{-1}
$$

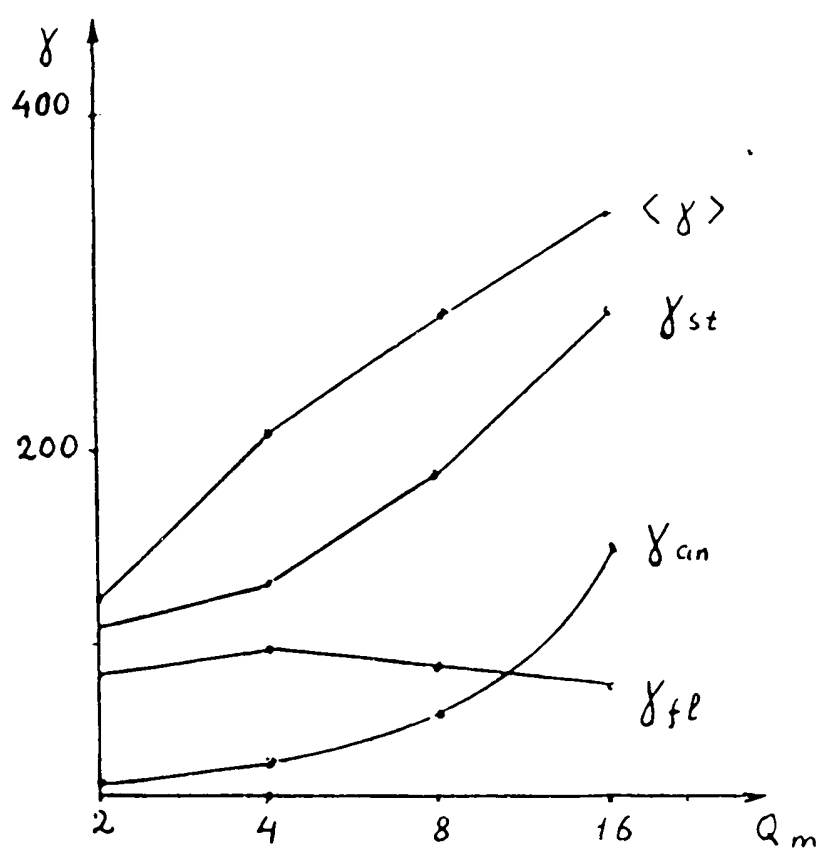

Figure 3 The electron Lorentz factor as a function of the extracting charge $Q_{\mathbf{m}}$.

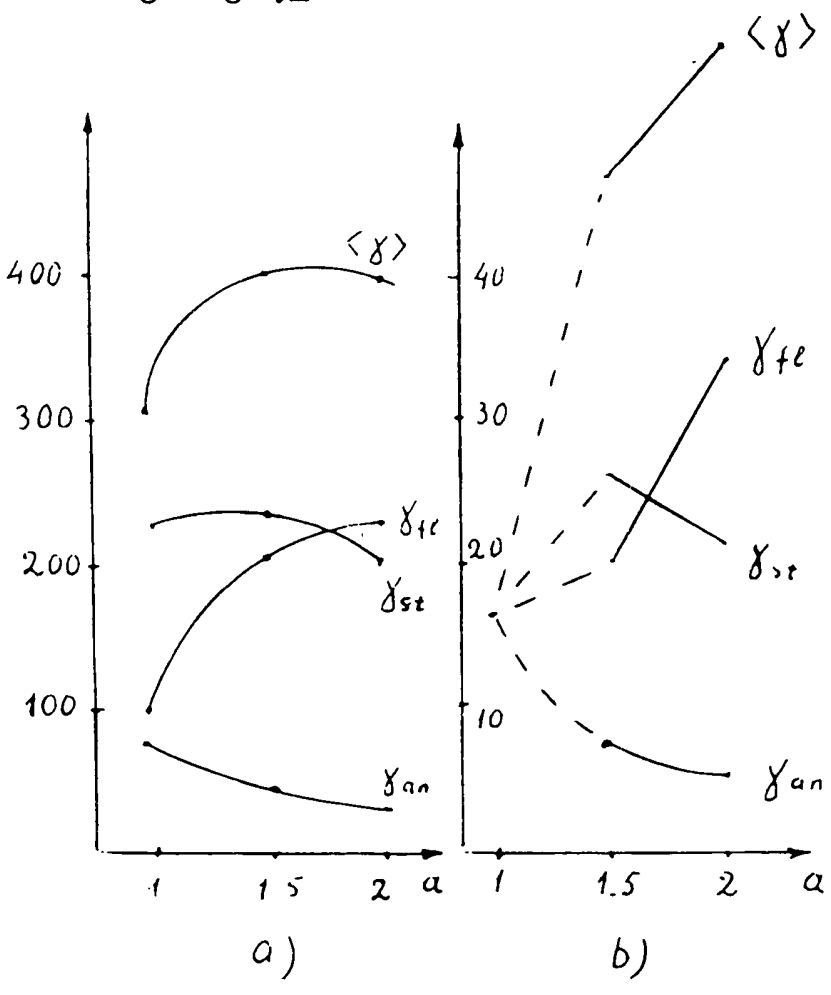

Figure 4 The electron Lorentz factor as a function of magnetosphere altitude $a=\left(R_{0}-R\right) / R,(a)$ for $e / m=100$, (b) for $e / m=10$.

\section{A model of the pulsar radio emission mechanism}

Estimates show that practically all the thermal energy of the electron-bunch gas converts into the radio emission (Rylov 1987). The pulsar magnetosphere is optically thick for radio emission. A model of the electron-bunch gas thus arises (Rylov 1982). 
There are three principal points to make about this model.

1. The temperature $k T$ of the bunch gas can be estimated as

$$
k T \approx m c^{2}(\gamma-1) n V,
$$

where $V$ is the bunch volume, $m, n$, and $\gamma$ are respectively the electron mass, the electron concentration, and the bunch Lorentz factor.

For $n \approx 10^{12} \mathrm{~cm}^{-3}$ and $V \approx 10^{6} \mathrm{~cm}^{3}$, the factor $n V \approx 10^{18}$. The temperature of the electron-bunch gas is $n V \approx 10^{18}$ times as large as that of the electron gas (if the electron Lorentz factor and the bunch Lorentz factor are of the same order). As long as the radio emission is emitted mainly by bunches, the brightness temperature of the radio emission is very high. It increases by a factor $n V$ as compared with the electron-gas radio emission. As a result the mechanism of radio emission appears to be coherent (many electrons of the bunch radiate in the phase) and thermal (bunches move chaotically) at the same timie.
2. The elastic scattering of the radio emission quanta is non-isotropic, because the electron bunches cannot move across the magnetic field lines. Thus the elastic scattering length of radio waves is very small along all directions except the direction along the magnetic field. Thus, the radio emission propagates only in the magnetic field direction. This explains the very sharp directivity of the pulsar radio emission (Rylov 1982).

3. There is some maximal size $L_{\max }$ of elecrron bunches which is conditioned by the outflow channel geometry. Estimation of the radio spectrum leads to the expressions (Rylov 1982)

$$
\begin{aligned}
& \rho(\nu)=A\left(\frac{\nu}{\nu_{0}}\right)^{2}, \quad \nu<\nu_{0} \\
& \rho(\nu)=A\left(\frac{\nu_{0}}{\nu}\right), \quad \nu>\nu_{0}
\end{aligned}
$$

where $\nu_{0}=c L_{\max }$. The observable low frequency turnover of the radio spectrum at $10^{8} \mathrm{~Hz}$ corresponds to the maximal bunch size $L_{\max } \approx 10^{2.5} \mathrm{~cm}$. This correlates with the width of the internal acceleration zone (Rylov 1979). 